\title{
Quantifying network flexibility requirements in terms of energy storage
}

\author{
Richard Nayak-Luke ${ }^{\mathrm{a}}$, René Bañares-Alcántara ${ }^{\mathrm{a}^{*}}$, Sam Collier ${ }^{\mathrm{a}}$ \\ ${ }^{a}$ University of Oxford, Department of Engineering Science, Parks Road, Oxford OX1 3PJ
}

\begin{abstract}
The energy storage requirement modelling tool outlined here uses an extension of the pinch methodology to quantify the network flexibility required due to greater variable renewable energy (VRE) integration. Quantifying this as the magnitude of energy storage required as a function of storage duration provides improved understanding of the fundamental balancing problem. Identification of the impact that key decision variables, such as location, VRE penetration and VRE that is supplied through wind have on these requirements has been enabled by condensing this dependence within two non-dimensional indices: the storage magnitude index and the storage duration index. These indices, in addition to the size of storage required, have enabled the tipping-points and VRE penetration levels beyond which short-term storage is not sufficient to be identified for the UK and Australian locations considered. In addition to how effective the provision of excess VRE is in the mitigation of the balancing requirements. This analysis shows that seasonal balancing is required at high levels of VRE penetration for all combinations of wind and solar sources, but can be needed with as low as 50\% penetration at certain combinations and locations. The tipping-point is highly specific to location and VRE that is supplied through wind, varying between $30 \%$ and 50\% of VRE penetration.

Keywords: Energy storage; Energy system modelling; Integration of renewables; UK; Australia.
\end{abstract}

\section{Highlights}

- Description of the Energy Storage Requirement Modelling Tool

- Quantification of the flexibility required for VRE integration in terms of energy storage magnitude and duration

- Identification of the tipping-point beyond which short-term storage is not sufficient

- Quantification of the mitigating impact of excess VRE supply

Number of words: 4,630 (excluding captions and references)

\footnotetext{
* Author to whom all correspondence should be addressed (rene.banares@eng.ox.ac.uk)
} 
Decarbonisation of power networks is a key objective for virtually all countries if they are to achieve their intended nationally determined contributions (INDCs) of carbon emissions. To achieve net-zero greenhouse gas (GHG) emissions it is essential to decarbonise the power sector (a key GHG emitting sector), and to facilitate the electrification, and thereby decarbonisation, of other GHG emitting sectors (such as transport, heating and industry).

To facilitate this necessary increase in variable renewable energy additional network flexibility is required. This requirement, however, is different for each local, regional, and national power network as shown in Figure 1. The reason for these differences is due to many factors, including the available VRE sources, demand, existing capacity and infrastructure, and available methods of flexibility. Planning and delivering the network flexibility required to facilitate the once in history transition from fossil-fuels to renewable power sources is an economic imperative [1].

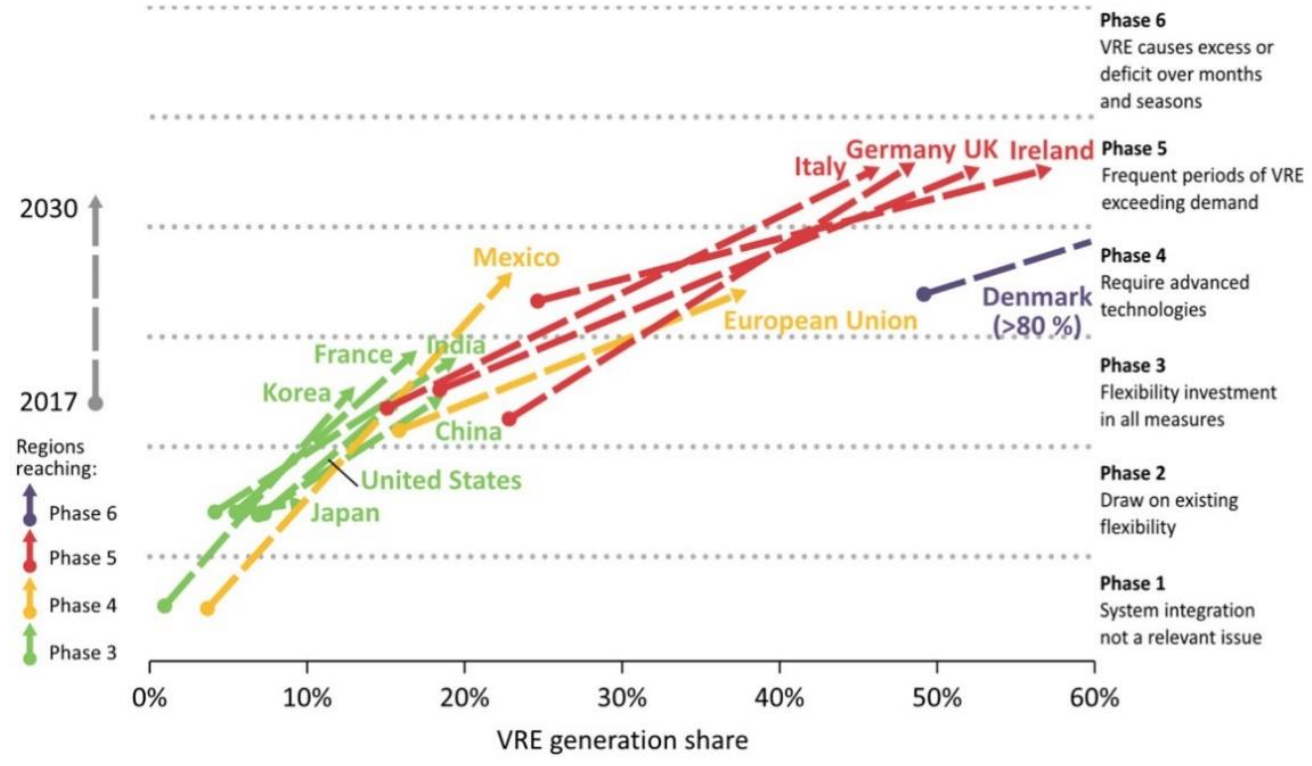

Figure 1:The IEA's prediction (in their New Policies Scenario) of future network flexibility requirements by country and variable renewable energy (VRE) penetration [2]

Previous work on this area can be divided into technical and techno-economic modelling. Techno-economic analyses require the user to find a balance between details and scope [3] in the selection and use of a tool while accounting for the limitations in modelling techniques and computing power [3] and an appreciation that no energy tool addresses all issues related to renewable energy integration [4]. There is a considerable amount of literature in this field and the use of these models can be classified depending on whether they consider technical, economic or 
market optimisation [5]. Zerrahn and Schill [6] have performed a review of those techno-economic models focussed on energy storage. Technical analyses on the other hand quantify the network flexibility requirement (i.e. the solving of the balancing problem). These analyses, beyond those only considering the optimal alignment of power profiles 48 [7], can be grouped by the type of flexibility method employed. Mitigating methods, such as interconnection or excess 49 capacity (i.e. allowing for curtailment) [8,9] reduce the occurrence and / or magnitude of demand exceeding supply 50 (i.e. deficits). Managing methods, such as demand-side management [10], reserves [11, 12] and energy storage [1351 18], subsequently eliminate remaining power deficits. Careful design of balancing the flexibility requirements with 52 methods is essential [19]. The economically optimal solution will likely be a mix of these options [20].

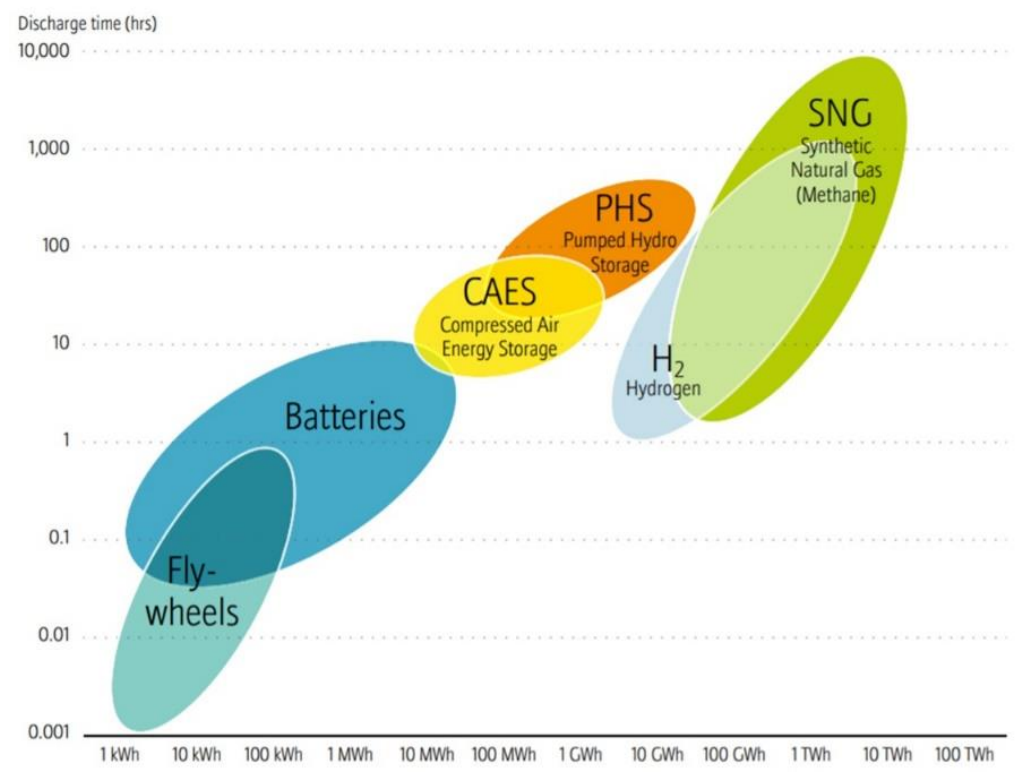

Figure 2: Energy storage capacity and discharge time of different energy storage methods [21]

There is a wide range of available energy storage technologies currently available that vary considerably in their technical characteristics (Figure 2) and cost. Further detail on the technical and economic of storage can be found in [22-24]. Implementation of energy storage to date has been predominantly short-term methods (such as batteries) that are able to competitively provide the quick response services (e.g. frequency response) that have been increasingly required due to VRE integration. As VRE penetration increases additional network flexibility will be required to 61 manage the intraday, interday and even seasonal balancing (Figure 1). Understanding how this requirement will develop for individual networks and planning so that it can be met cost effectively is important. 


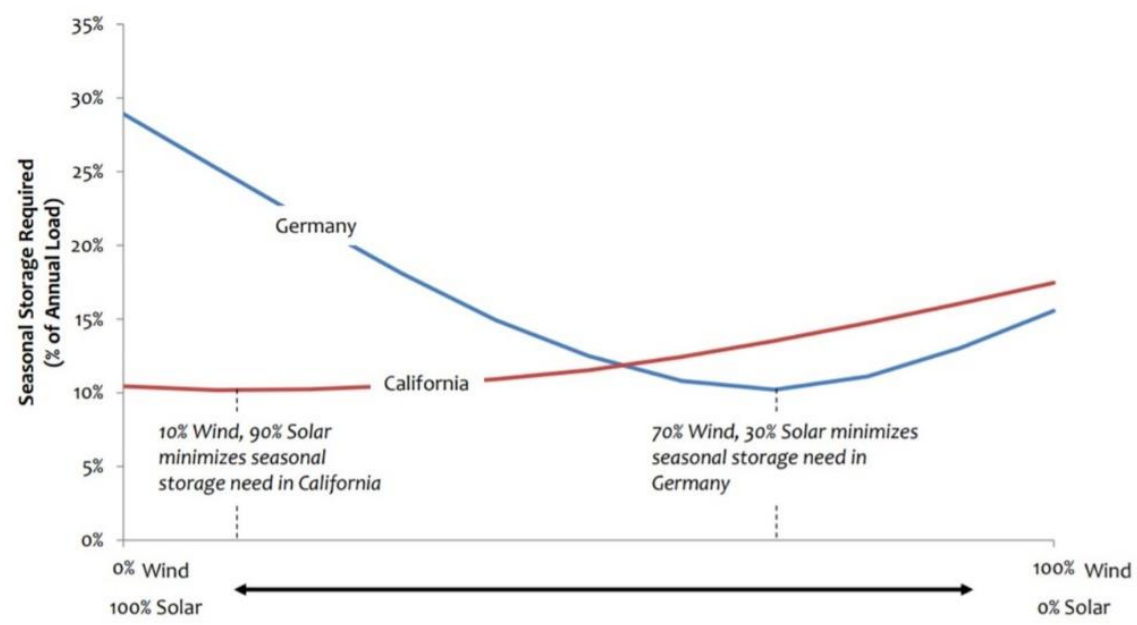

Figure 3: Seasonal storage required as a function of the VRE that is supplied through wind met by wind for Germany and California at 100\% VRE penetration [17].

Previous investigations quantifying a network's flexibility requirement primarily in terms of energy storage have focussed on the magnitude of energy that needs to be stored $[14,17,25,26]$ and on how this can be mitigated by careful selection of the mix of VRE sources [15]. Analysis on the temporal aspect (i.e. storage duration) of the flexibility requirement as VRE is integrated has been limited to the consideration of single [25] or a limited range of duration (as shown in Figure 3) [17, 26, 27]. To identify the viable design space for an energy system the pinch methodology [28] can be used. This method, which was originally developed to consider the optimisation of heat recovery in chemical processes, has been extended to systematically find the optimal exchange of resources [29], energy or mass [30, 31], for a given objective function. An example of the application of pinch methodology considering energy, although limited to battery storage, is presented in Bandyopadhyay [32]. This paper, building upon previous work [33], demonstrates the methodology and initial results of the energy storage requirement modelling tool (ESRMT) that quantifies a network's balancing problem temporally as well as its magnitude by calculating the energy storage required using a modified pinch methodology that matches energy sources and sinks temporally. Focussing on hourly to intra-year balancing requirements, the use of the ESRMT has enabled the identification of the VRE penetration at which the storage required cannot be met by short-term storage alone (i.e. a tipping point) by location and the mix of VRE sources employed. Results, when considering the use of multiple flexibility methods, provide a technically viable domain for techno-economic optimisation. Quantitative estimation of these tipping points is important to better understand how network's flexibility requirements will develop with greater 
VRE integration, how to increase the VRE penetration at which they occur, and the subsequent mix of balancing 85 requirements after they are surpassed.

86 This paper is organised as follows: Section 2 describes the methodology of the Energy Storage Requirement 87 Modelling Tool (ESRMT), Section 3 outlines the calculations performed, Section 4 analyses the results, and Section 885 outlines the key conclusions and scope for future work.

\subsection{Overview: Inputs, outputs and assumptions}

The ESRMT is a tool, written in MATLAB, which can solve quantitatively the fundamental technical problem of balancing electrical supply and demand as a function of the energy storage required. The requirement itself is required duration of storage $(\tau)$ (its short- / long-term bias). Inputs to the ESRMT include time synchronous supply and demand energy vectors, a set of assumptions, and control panel inputs such as the decision variables to be considered. Output from the ESRMT is primarily based on the calculated storage profile: the amount of energy that has to be stored to meet demand as a function of storage duration (as shown in Figure 4). To quantitatively identify the impact of any given decision variable(s) on the storage requirements, two non-dimensional normalised indices are calculated: the storage magnitude index (SMI) and the storage duration index (SDI). The other output from the ESRMT is the capacity (as a percentage of mean daily energy demand) of the storage required for user-defined ranges of storage duration $(\tau)$. In this paper four storage duration groupings have been considered:1 hour to 1 day, 1 day to 1 week, 1 week to 1 month and 1 month to 1 year. The ESRMT assumes (i) perfect foresight of all future energy supply and demand, (ii) dispatchable energy to be perfectly flexible (i.e. no ramping limitations), (iii) dispatchable energy has no upper limit (i.e. the dispatchable supply rated power is not specified), (iv) the storage can charge and discharge its entire energy capacity in any given hour, and (v) the storage has no losses. These assumptions ensure that the calculated storage requirements are as small and as short-term biased that they can be for the islanded power network considered, thereby providing an initial quantitative assessment of the fundamental balancing problem that needs to be managed. Relaxation of these assumptions, as suggested later as future work, will increase the storage magnitude and duration. Comparison of results with and without some/all of these assumptions, in addition to other 
factors such as interconnection, will enable the quantification of their impact on the fundamental balancing problem.

111 The ESRMT is fundamentally a pinch methodology which identifies the optimal schedule and magnitude of storage 112 which minimises the duration of stored energy. This is achieved by identifying the maximum duration of storage required for each energy deficit interval to be filled, and then considering deficit intervals sequentially. A worked example using the methodology can be found in the Supplementary Material (Error! Reference source not found., Error! Reference source not found. and Tables 3-7).

\subsection{Detailed description of the model}

\subsubsection{Step 1: Control panel and energy vectors input}

The user provides the time-varying synchronous supply and demand energy data to consider. The entire sequence of values specified in this paper as $W, S$ and $L$ for wind, solar photovoltaic and demand are $\left(t_{\text {end }} \times 1\right)$ vectors respectively. The integral of each of these, and other vectors, over the time period of data provided $\left(1 \leq t \leq t_{\text {end }}\right)$ is shown by edged brackets (e.g. $\langle W\rangle$ is the total amount of energy provided by the wind power source). The tool's control panel allows the user to specify the decision variables to consider such as the: VRE penetration (Equation 1), VRE that is supplied through wind (Equation 2), and /or the excess energy factor (Equation 3, where $D$ is the dispatchable energy vector to be determined in Step 5). The user must specify either a single objective function or multiple objective functions for the allocation of dispatchable energy. The single objective functions are to either minimise the maximum storage duration required, or minimise the maximum energy stored or discharged in any given time interval (shown in Equation 4 respectively). Alternatively, if an attribute such as the rated charging power, rated discharging power, or capacity of the storage technologies is being considered, multiple objective functions and their priority are required (shown in Equation 5 respectively, where $I$ is the energy vector for energy stored and $O$ is the energy vector for energy discharged from storage). If selected, these multiple objective functions are prioritised by the user. They are considered sequentially, moving onto the next when the objective function has been satisfied (e.g. the capacity of long-term storage being less than $40 \mathrm{MWh}$ ) by the allocation of available dispatchable energy, or stopping if the available dispatchable energy equals zero.

$$
\text { VRE penetration }=\frac{\langle W\rangle+\langle S\rangle}{\langle W\rangle+\langle S\rangle+\langle D\rangle}
$$

$$
\text { VRE that is supplied through wind }=\frac{\langle W\rangle}{\langle W\rangle+\langle S\rangle}
$$




$$
\begin{aligned}
& \text { Excess energy factor }=1-\frac{\langle W\rangle+\langle S\rangle+\langle D\rangle}{\langle L\rangle} \\
& \text { Single objective function to minimise }\left\{\begin{array}{l}
\tau_{\max } \\
I_{\max } \\
O_{\max }
\end{array}\right.
\end{aligned}
$$

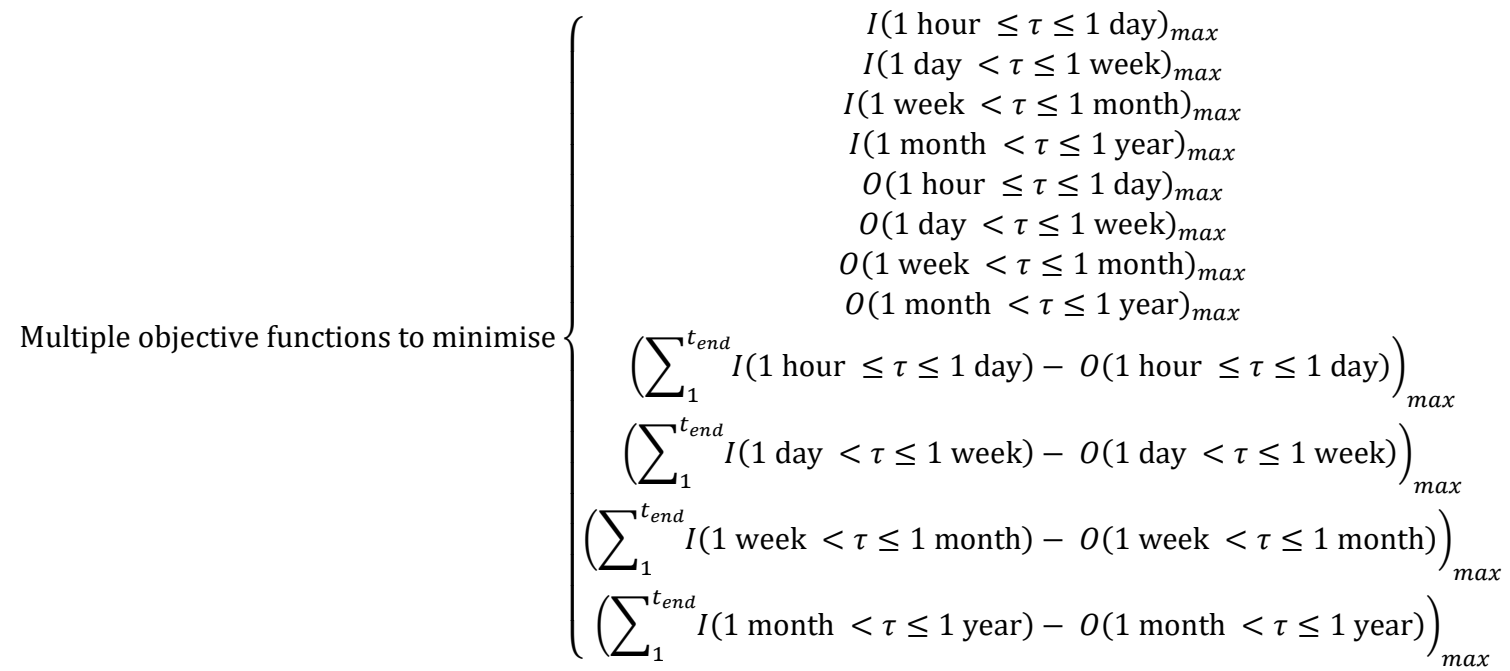

\subsubsection{Step 2: Calculation of the delta vector $(\Delta)$}

The wind and solar PV energy vectors are scaled according to the control panel inputs, i.e. the defined scenario to consider. The summation of these vectors and subtraction of the demand results in the delta vector ( $\Delta$ in Equation 6 ), 142 the balancing problem to be solved.

$$
\Delta=W+S-L
$$

\subsubsection{Step 3: Prioritisation of deficits}

To mitigate the balancing problem the available dispatchable energy needs to be allocated. However, before this can happen, one needs to determine how it should be allocated (i.e. which deficits should be prioritised). First, the maximum storage duration (i.e. the maximum amount of time required for a surplus to be shifted to fill a deficit) required for each time interval with an energy deficit is calculated. This, combined with the user defined objective function in the control panel, enables the deficits to be ranked and thus the order in which the intervals are considered and the allocated energy if available. For the remainder of this paper, and the worked example in the Supplementary 
150 Material, the minimisation of the maximum storage duration has been used as the objective function so that shorter151 term storage is preferred over longer-term alternatives.

\subsubsection{Step 4: Population of the storage matrix}

153 Considering each of the remaining deficits in turn, the storage matrix (a record of the magnitude and storage 154 duration of every surplus energy shifted to a deficit) is populated considering the deficit time intervals sequentially. 155 Each row is an occurrence of energy being stored to fill a deficit and it consists of the surplus index, the duration of 156 storage, the amount of energy shifted and the deficit index. The surplus and deficit indices are the time intervals from 157 which energy is stored and provided respectively.

\subsubsection{Step 5: Allocation of available dispatchable energy}

To appropriately allocate the dispatchable energy, the storage matrix is sorted in accordance with the technical objective function defined in the control panel (e.g. if the minimisation of storage duration is selected, then the matrix is sorted by the storage duration). The available dispatchable energy $(\langle\mathrm{D}\rangle)$ is allocated using the sorted storage matrix until either there is no more available energy to allocate, or there are no more deficits to fill (i.e. the storage matrix is empty).

\subsubsection{Step 6: Calculation of the storage profile}

The remaining storage matrix is sorted by storage duration. The cumulative sum of the energy column by storage duration determines the amount of energy stored for that duration or less. Normalising this vector using the total energy demand $(\langle\mathrm{L}\rangle)$ results in the storage profile for that simulation (shown in Figure 4): a vector $\left(\mathrm{E}_{\tau}\right)$ that is the fraction of demand met by stored energy as a function of the storage duration $(\tau)$. Storage requirements that exceed a 169 year are determined, however they are not considered in the calculation of the SMI and SDI. 


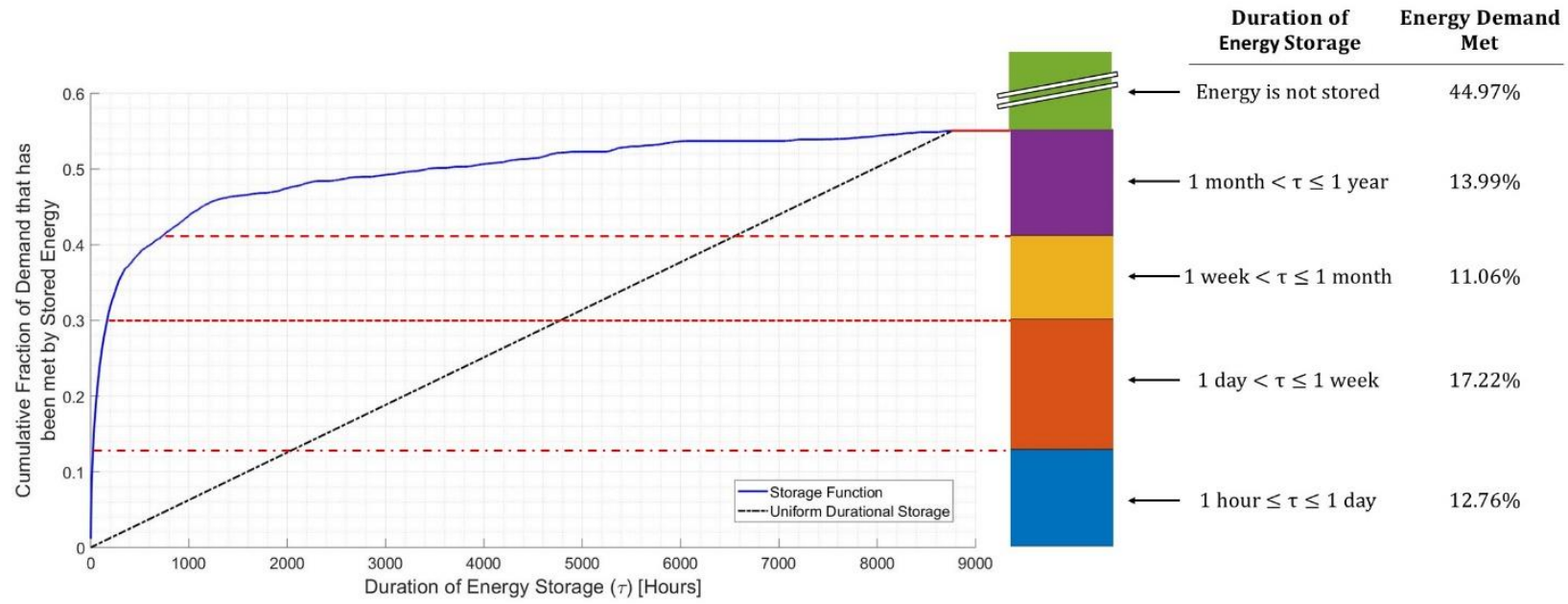

Figure 4: The' storage profile' for Strathallan Airfield, Perthshire, UK (January 2015-December 2019) with 100\% VRE penetration, 100\% Wind:0\% Solar PV power supply, no VRE curtailment, no DSM, and no interconnection.

Despite the storage profile being a very useful result on its own from which many insights can be gleaned (e.g. the

relative dependence on storage as a function of its duration as shown in Figure 5), comparing even a small number of storage profiles is difficult and not quantitative. This therefore limits the usefulness of such an analysis as the impact of key variables (such as VRE penetration, VRE that is supplied through wind, excess capacity and the incorporation of flexibility methods such as DSM) on the storage requirements cannot be identified or quantified.

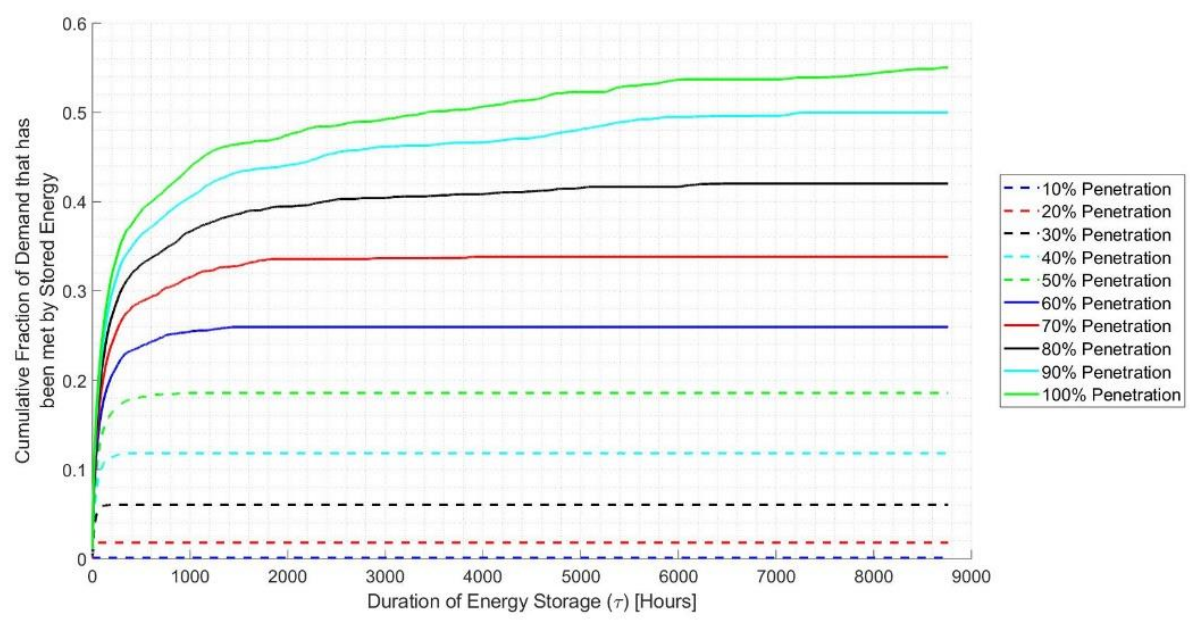

Figure 5: The difficulty in comparing storage profiles. Storage profiles for Strathallan Airfield, Perthshire, UK (January 2015 - 


\subsubsection{Step 7: Calculation of the SMI, SDI and size of storage}

To enable the storage requirements to be quickly identified and thus determine the impact of decision variables, the information in the storage profile is condensed into two non-dimensional numbers that quantify the magnitude and the durational bias of the storage requirements: the storage magnitude index (SMI), and the storage duration index (SDI). These are calculated from the storage profile using Equations 7 and 8 (shown graphically in the Supplementary

Material, Error! Reference source not found. and Error! Reference source not found.), where $T$ is the maximum storage duration considered in the SMI and SDI calculations, defined here as 8,760 hours.

$$
\mathrm{SMI}=E_{T}
$$

$$
\mathrm{SDI}=\frac{\int_{0}^{T}\left|E_{\tau}-\left(\frac{\tau}{T} \cdot E_{T}\right)\right| d \tau}{\frac{T \cdot E_{T}}{2}}
$$

The SMI, as shown in Figure 6 considering the variation of VRE penetration and the mix of VRE sources, enables an easy comparison of the impact of decision variables on the magnitude of storage required. Point 1 in Figure 6 and Figure 7 is for $100 \%$ VRE penetration, $50 \%$ of the energy supplied provided by wind sources and the other $50 \%$ by solar PV. Point 2 is for 50\% VRE penetration, 50\% of the energy supplied by wind sources, $0 \%$ from solar PV and the other 50\% from dispatchable resources. Point 1 has an SMI of 0.478 and Point 2 of 0.186. Showing, as expected,

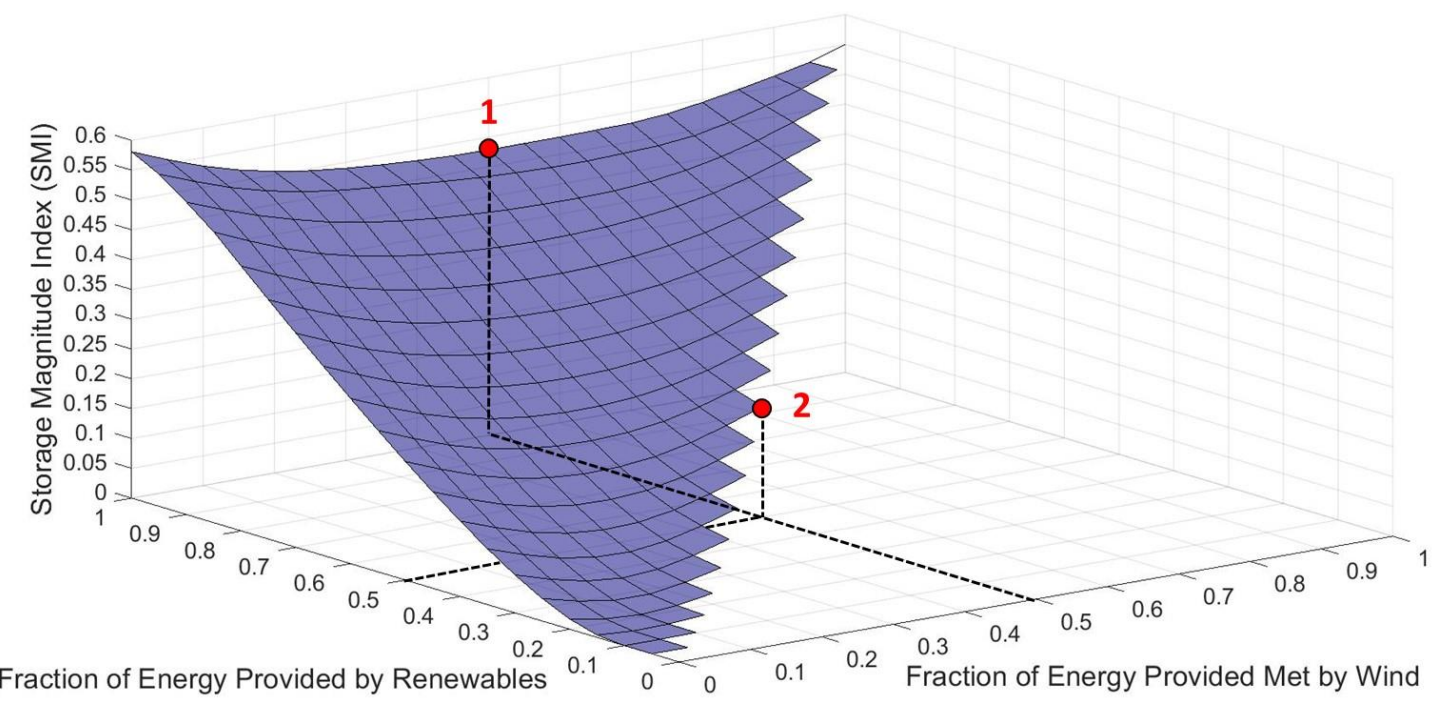


The SDI, as shown in Figure 7, highlights the impact that decision variables have on the mix of storage required to

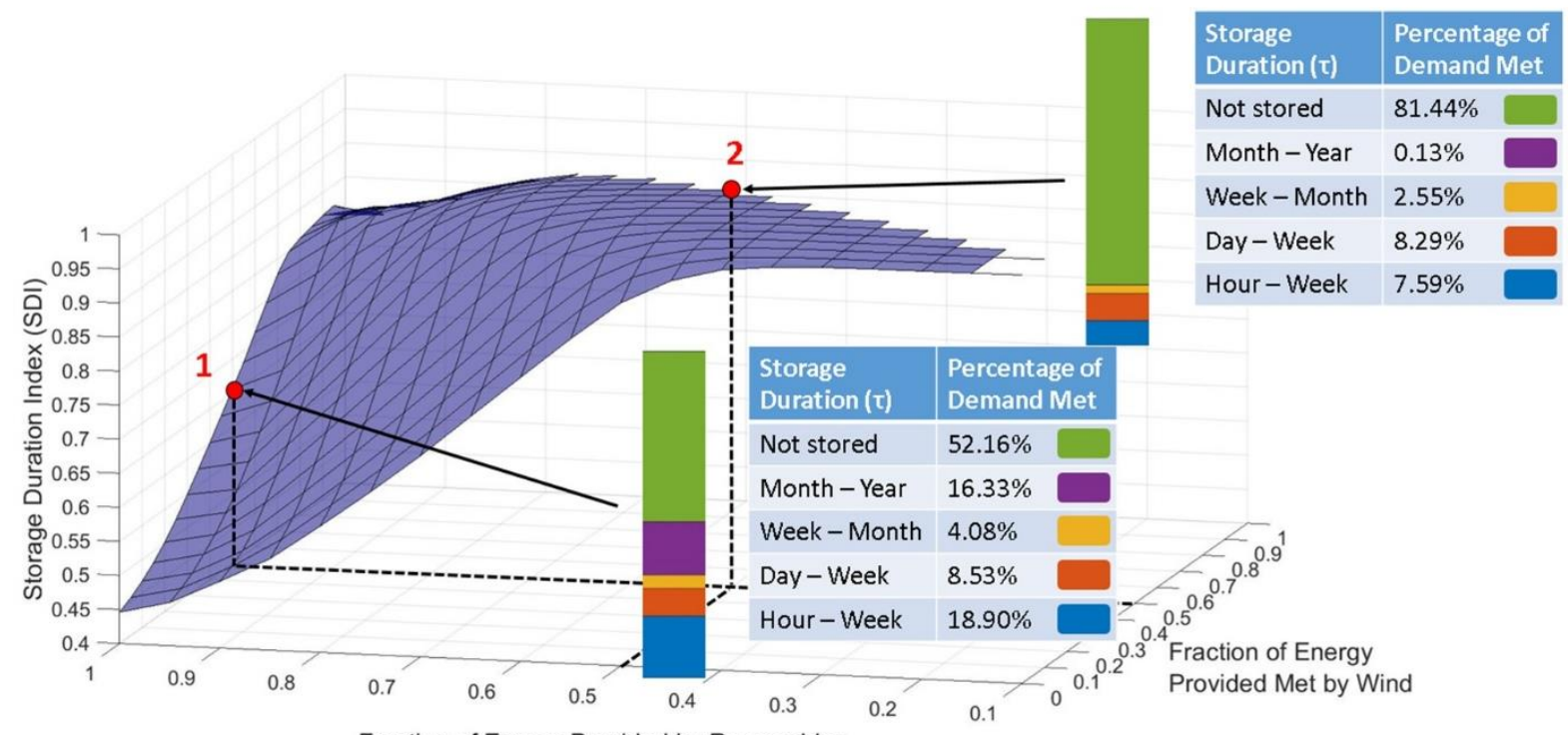

Figure 7: Impact of VRE penetration and the mix of VRE sources on the SDI of Strathallan Airfield, Perthshire, UK (January 2015 - December 2019). No excess energy supply and no other flexibility methods are employed.

Material, Error! Reference source not found.): 1 if the storage demand can be met solely by 1-hour storage (a hypothetical storage method that can only store energy for a duration of one hour), 0 if it is (on average) equally dependent on all durations of storage, and -1 if it can be met solely by 8760 -hour storage (a hypothetical storage method that can store energy for a duration of 8760 hours).

\section{Calculations}

To achieve our aim of identifying the impact that key variables such as location, VRE penetration, VRE that is supplied through wind and excess capacity have on the storage requirements of a system we considered 14 locations in the UK and 23 in Australia. The ESRMT was run using 2000-2020 Met Office wind and solar MIDAS data [34] for the UK, and Meteonorm [35] wind and solar data for Australia both with hourly resolution. The wind speed provided in the weather data was first converted to a hub height of $80 \mathrm{~m}$ using Equation 9 (where $\mathrm{U}$ is wind speed 
$(\mathrm{m} / \mathrm{s}), \mathrm{H}$ is height $(\mathrm{m})$, and $\mathrm{z}$ is roughness length $(0.03 \mathrm{~m}))$, then converted to power using the power profile of the

Vestas V90 3.0 MW turbine with an air density of $1.225 \mathrm{~kg} / \mathrm{m}^{3}$ [36]. The cut-in and cut-out speeds were taken as 4 and $25 \mathrm{~m} / \mathrm{s}$ respectively.

$$
U_{1}=U_{2}\left(\frac{\ln \frac{H_{1}}{Z}}{\ln \frac{H_{2}}{Z}}\right)
$$

The demand was taken as ELEXON profile class 1 [37] for the UK's locations, and 2015-2020 state network demand for Australia [38]. Using these inputs, the SMI, SDI, storage use and storage size was calculated for each location. Limited by the availability of demand data, a time period of five years has been used so that locations in different countries can be compared. The impact of the time period of the data on the SMI, SDI, storage use and size is shown in the Supplementary Material.
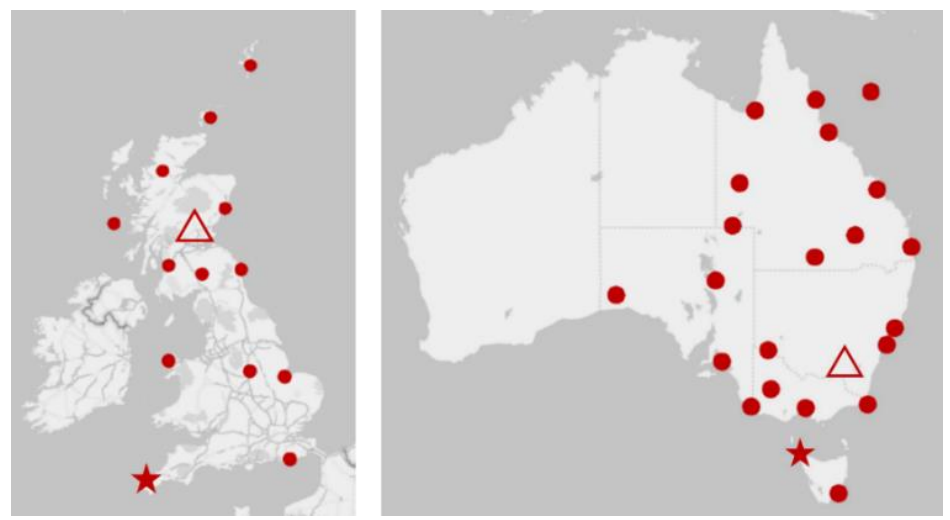

Figure 8: Locations assessed using the ESRMT. See Supplementary Material, Error! Reference source not found. and Error! Reference source not found. for specific locations. Triangle and starred locations are examples of the more and less challenging locations considered for that country respectively.

\section{Results}

\subsection{Identification of the requirement tipping-points (i.e. when short-term storage is not independently sufficient)}

Calculation of the SMI for each of the locations considered and the identification of the maximum and minimum

SMI by country for each combination of decision variables (VRE penetration and VRE that is supplied through wind) enables the comparison of the magnitude of storage required (Figure 9). As expected, the magnitude of storage required increases markedly with VRE penetration. Furthermore, it is also impacted by the VRE that is supplied through wind, and an appropriate combination of the VRE sources can minimise the magnitude of storage 
required. However, for both the UK and Australia the maximum and minimum values of the SMI for the sites considered are highly dependent on location, particularly at high VRE penetration.
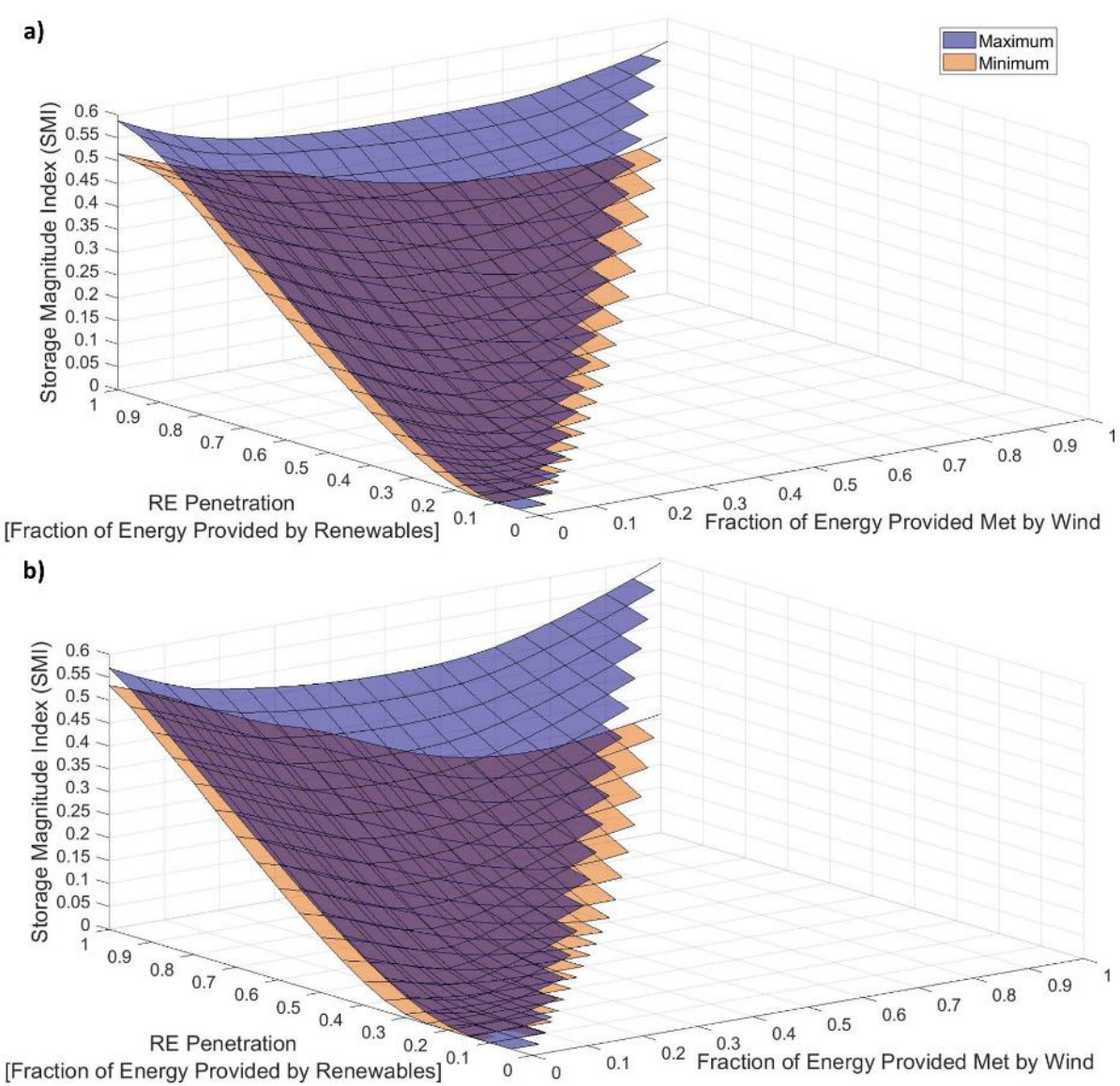

Figure 9: Impact of VRE penetration, the mix of VRE sources, and the location within (a) the UK and (b) Australia on the SMI.

The maximum requirements for each country, despite having some differences at low VRE penetration, have notable similarities such as the requirement at high VRE penetration and the relatively small impact of VRE that is supplied through wind. The SMI at 50\% VRE penetration in the UK is between 0.126 and 0.229 (i.e. a range of 0.103 ) and in Australia is between 0.096 and 0.200 (i.e. a range of 0.104 ). The SMI at 100\% VRE penetration in the UK is between 0.478 and 0.587 (i.e. a range 0.109) and in Australia is between 0.450 and 0.589 (i.e. a range of 0.139 ). Despite the SMI being similar at high VRE penetration for solar dominated systems and the integration of wind in the VRE that is supplied through wind reducing the SMI (for a given VRE penetration), the minimum requirement in each country have many differences. Arguably the most significant of these are the VRE penetration at which the SMI 

VRE penetrations.

a)

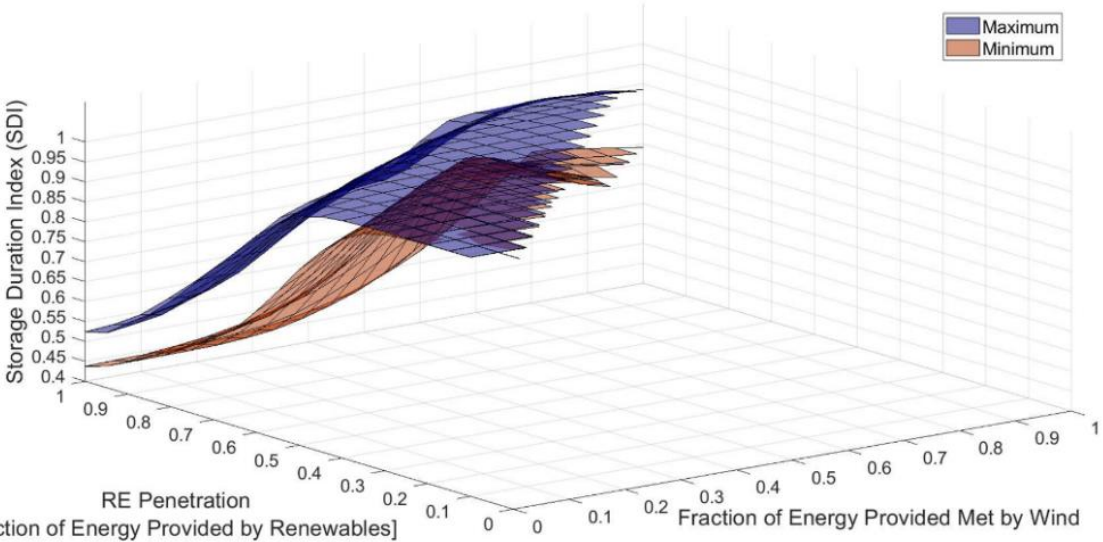
[Fraction of Energy Provided by Renewables]

b)

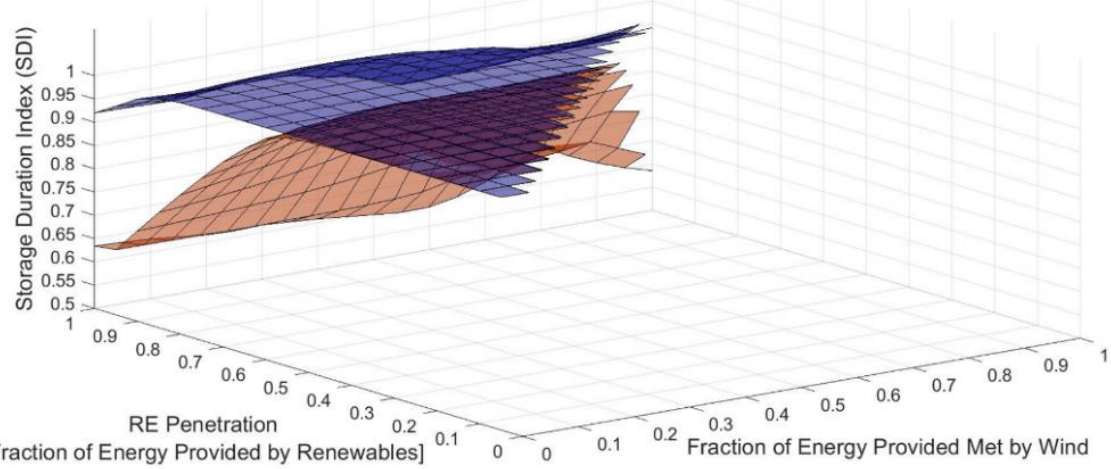

Figure 10: Impact of VRE penetration, the mix of VRE sources, and the location within (a) the $U K$ and (b) Australia on the SDI.

The comparison of the SDI calculations shows, as expected, that as VRE penetration increases the storage requirement cannot be met by short-term storage technologies alone. More specifically, as VRE penetration increases the storage requirements are less short-term bias and the SDI can be used to quantify the tipping point at which shortterm storage is not sufficient to balance supply and demand (when the SDI is not approximately equal to 1). As shown in Figure 10, the VRE penetration tipping point varies significantly with the VRE that is supplied through wind, location and country. An example of this can be observed when considering the maximum and minimum SDI in both countries for solar dominated systems. In Australia the SDI does not decrease below 0.990 (i.e. highly short-term storage biased, as shown in the Supplementary Material, Error! Reference source not found. and Error! Reference source not found.) until at least 55\% and most 90\% VRE penetration. Unsurprisingly, solar dominated systems reach 
reliance on longer-term storage increases) also varies by country. The locations in the UK that are highly dependent on solar have considerable medium to long-term balancing requirements at high VRE penetration, whereas many Australian locations do not. While not conceptually surprising, quantification of when these tipping points occur and how they develop with VRE penetration is of importance for policy decision-making. However, quantifying when the tipping point has been reached is difficult, primarily because there is not a universal consensus on what constitutes a tipping point. In this paper we have it as the bias of the storage requirements, i.e. the VRE penetration (Equation 1) $0.990,0.950$ or 0.900 , thereby identifying that the storage required cannot be entirely met by very short term energy storage methods. Alternatively, Figures 13, 14, 25 and 26 allow for a tipping point based on the magnitude of the required medium and/or long term storage to be identified. The tipping points based on the bias of the storage requirements for the results in Figure 10 are shown in Supplementary Material, note 5, tables 8-11.

The Australian locations considered are seen to be relatively insensitive to the VRE that is supplied through wind, therefore resulting in it not having a significant impact on when the VRE penetration tipping-point occurs. However, as shown in Figure 10a, this is not the case for UK locations where the VRE penetration at which the SDI decreases below 0.990 is highly sensitive to the VRE that is supplied through wind and location. In wind dominated systems the maximum SDI result does not fall below this level until 75\% VRE penetration.

The optimal VRE that is supplied through wind to minimise the storage requirement varies not only by location, but also by the objective function. These results show that the optimal VRE that is supplied through wind to minimise SMI is not necessarily the same as the VRE that is supplied through wind that minimises SDI. In other words, only considering the magnitude of storage required and ignoring its duration could lead to a solution unnecessarily dependent on longer-term storage methods for a minimal reduction in the magnitude. For example, at $100 \%$ VRE penetration the optimal VRE that is supplied through wind to minimise SMI is $50 \%$ (SMI $=0.478$ ). However, the duration of storage required (quantified as an SDI of 0.786) is considerably less short-term biased than for a VRE that is supplied through wind of $70 \%(\mathrm{SMI}=0.481$ and $\mathrm{SDI}=0.902)$.

\subsection{Mitigation of the storage requirements by providing excess supply}

Other flexibility methods can be included as additional decision variables to determine how they can mitigate the storage requirements. One such balancing problem mitigation method is the provision of excess VRE supply (i.e. 
Figure 11: Impact of VRE penetration, the mix of VRE sources, and the excess energy supplied on the SMI at (a) the least and (b) most challenging locations considered in the UK (2015-2020 Cambourne and Strathallan Airfield respectively).

allowing for curtailment of available variable renewable energy). Figure 11, Figure 12, Error! Reference source not
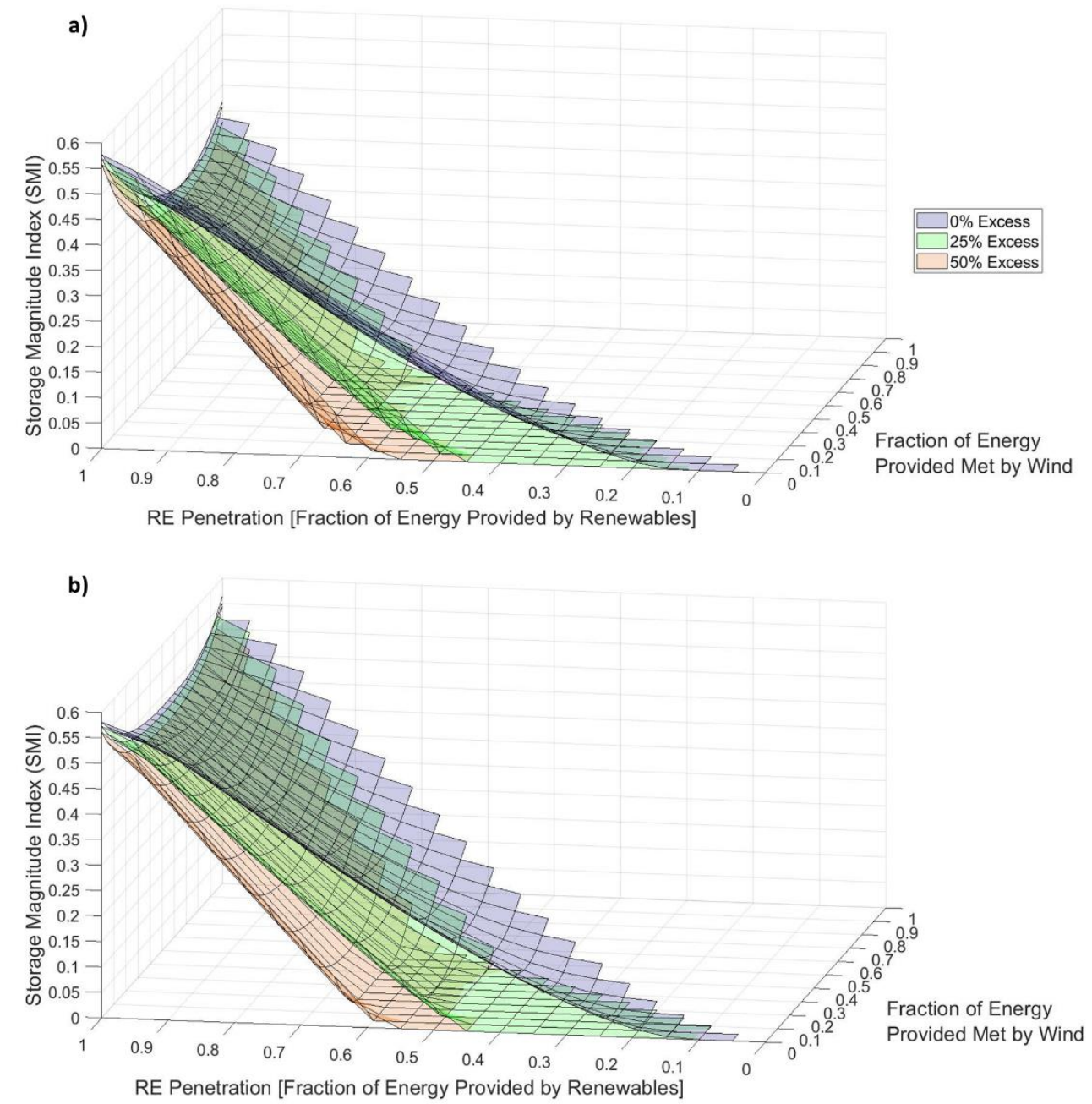

found. and Error! Reference source not found., show that the magnitude of the excess energy supply, depending mix of storage required. 


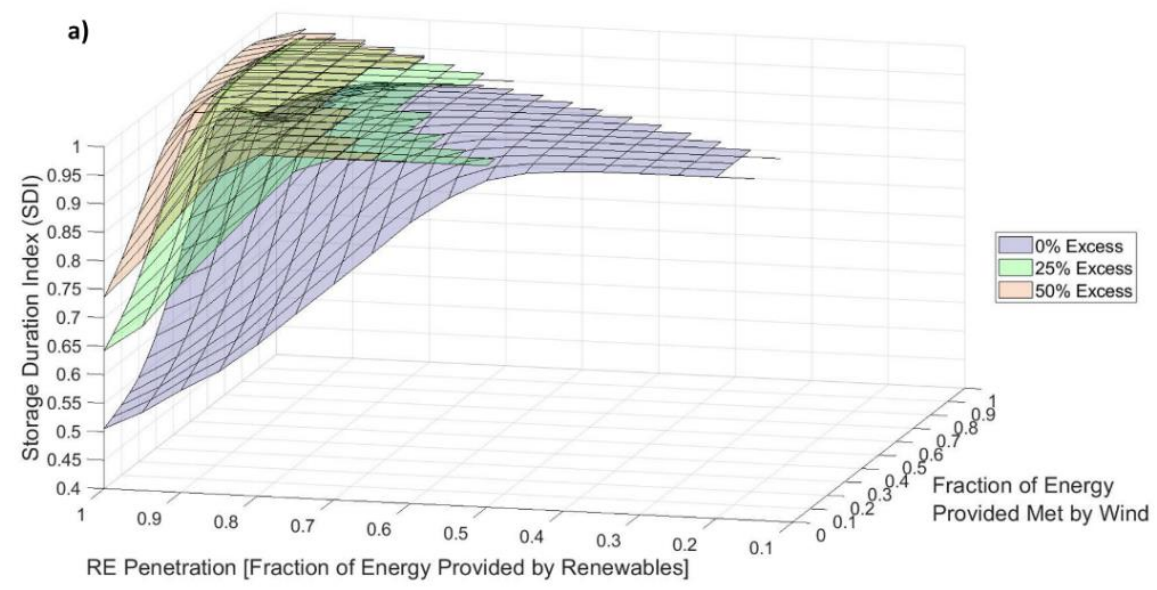

b)

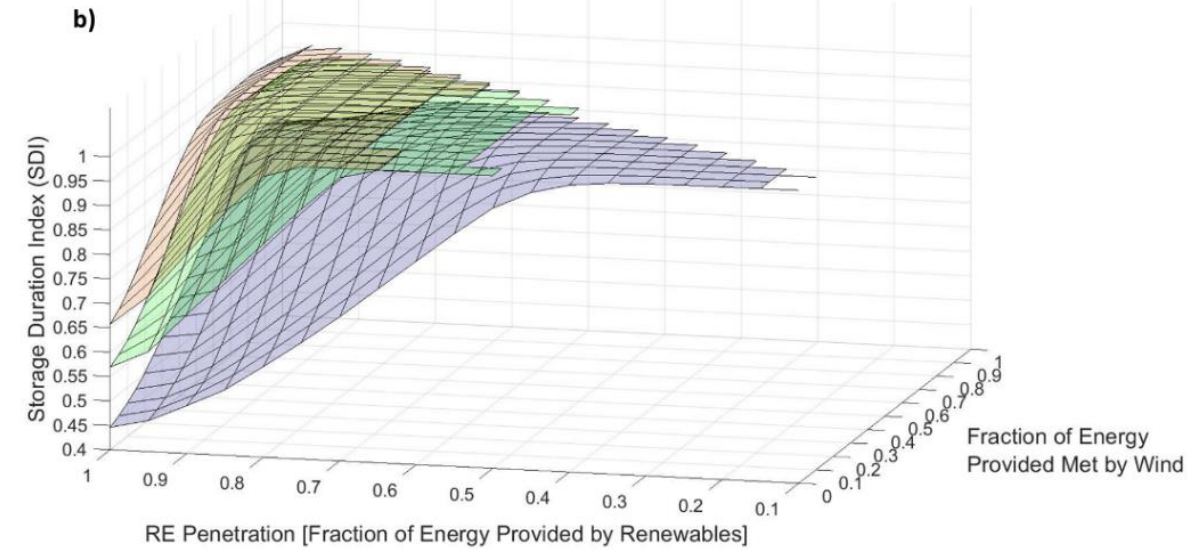

Figure 12: Impact of VRE penetration, the mix of VRE sources, and the excess energy supplied on the SDI at (a) the least and (b) most challenging locations considered in the UK (Cambourne and Strathallan Airfield respectively).

Comparing the impact that excess energy supply has on the SMI for one of the most and least challenging locations of those considered in the UK (Figure 11), shows that location can not only dictate the balancing problem, but also how difficult it is to mitigate. For example, the provision of $25 \%$ excess energy pushed back the initial requirement for energy storage in a solar dominated system to 40\% VRE penetration in the more challenging location (Figure 11b), but $45 \%$ less challenging location (Figure 11a). However, this is not the same for all values of VRE that is supplied through wind. The provision of excess supply with certain values of VRE that is supplied by wind that align better with the demand profile push back the initial requirement for energy storage a further 5\% VRE penetration relative to either wind or solar dominated systems for $25 \%$ excess supply. Providing $50 \%$ excess supply not only shifts the initial storage requirement to a higher VRE penetration, but also increases the discrepancy by VRE that is supplied through wind to $10 \%$ of VRE penetration. Nevertheless, as it can be seen in Figure 11, mitigation of the magnitude of energy storage requirements by excess capacity has its limitations. In both locations, the diminishing mitigation of the SMI 
can be seen when increasing the excess capacity. The extent of the mitigation at very high penetration levels (i.e. greater than or equal to $85 \%$ ) is relatively minimal. Similar trends were observed for the equivalent Australian locations considered (Supplementary Material, Error! Reference source not found.).

Importantly, providing excess energy not only mitigates the magnitude of storage required, but also delays (until

\subsection{Energy storage required: capacity and relative importance}

As expected, the size the storage correlates with the SMI. However, as shown in Figure 12a, the storage size required, particularly longer-term storage size at higher VRE penetration, can differ from what the storage use results could lead you to expect. For example, the total storage size required at $100 \%$ VRE penetration and $0 \%$ excess energy at Cambourne for $100 \%$ and 50\% VRE that is supplied through wind is considerably smaller than that required for $0 \%$ wind: $15.3,11.4$ and 30.3 days of average energy demand (with SMIs of $0.42,0.37$ and 0.58 ) respectively. The non-solar dominated systems, due to their shorter-term storage requirements, are able to utilise short-term storage 
methods hundreds of times a year to meet the balancing requirement, whereas the longer-term storage requirements 338 of the solar dominated system cannot.

339 The impact of excess supply to mitigate the storage requirements previously described can also be seen when 340 comparing Figure $12 \mathrm{a}$ with $12 \mathrm{~b}$ and $12 \mathrm{c}$, and Figure $13 \mathrm{a}$ with $13 \mathrm{~b}$ and $13 \mathrm{c}$. Particularly the ability of excess energy 341 to mitigate the storage requirements. The longer-term storage requirements can be seen to be difficult to eliminate 342 with excess energy supply, particularly in solar dominated systems in the UK. At very high VRE penetration, greater 343 than $80 \%$, the provision of excess energy (particularly in systems with energy from solar sources) can be seen to 344 only marginally reduce the overall size of storage required, but shifts the mix of required storage away from long345 term to other methods as was expected from the SMI and SDI results shown earlier.

Comparison of the size of storage required for the two UK locations considered (Figure 12 and 13) shows that while it differs in magnitude with respect to the VRE penetration, the trends are otherwise very similar. This is also the case for the Australian locations considered (Supplementary Material, Error! Reference source not found. and Error! Reference source not found.) except for the size of storage required (particularly for longer-term storage 1 month $\leq \tau \leq 1$ year) being smaller. This, as noted previously, is in large part due to the more favourable solar resource which reduces the medium to long-term balancing requirements. 
a)

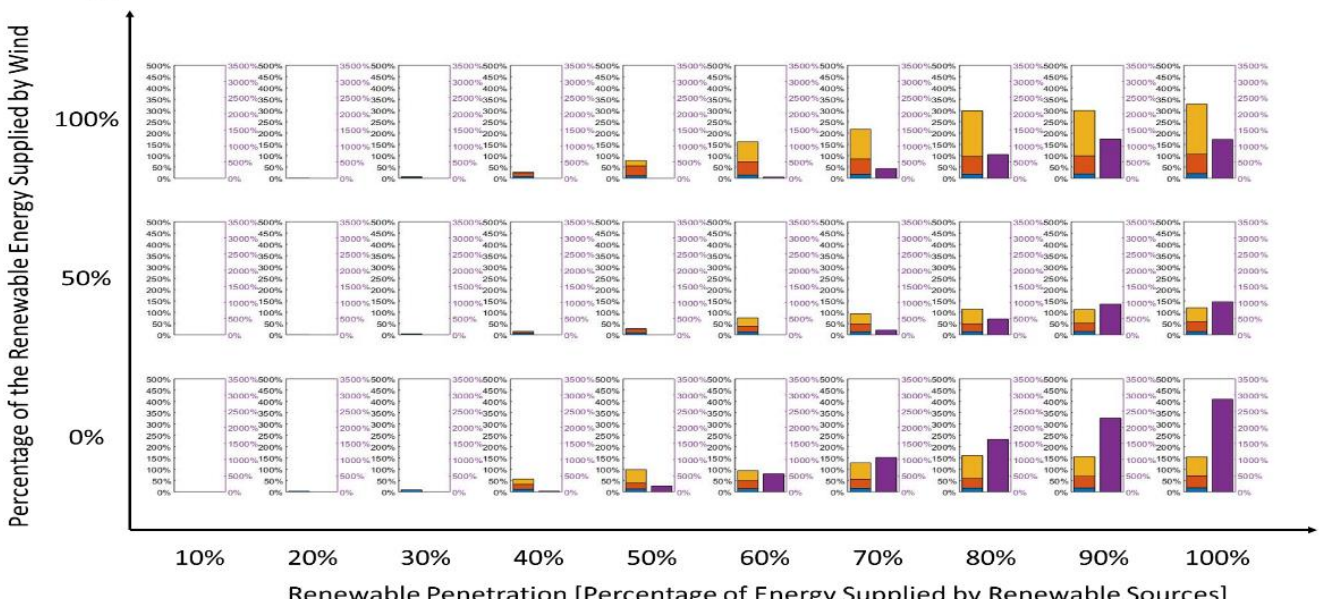

b)

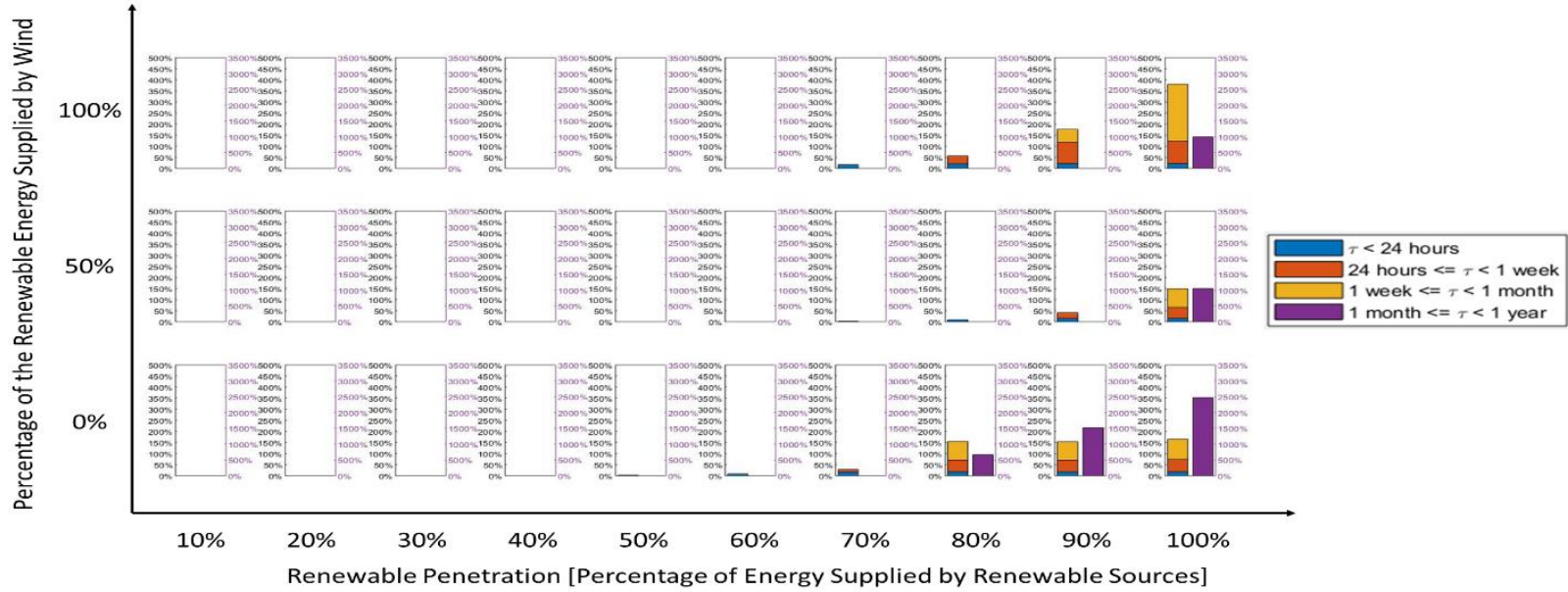

c)

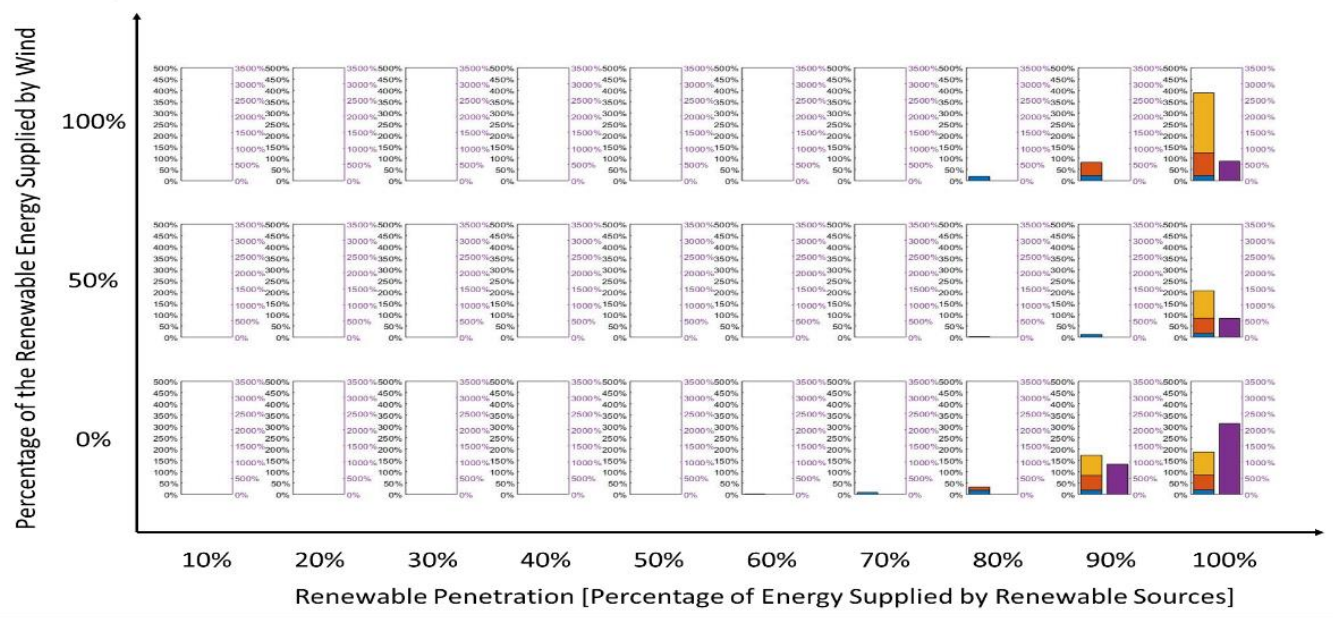

Figure 13: Least challenging location considered in the UK (Cambourne): Storage size required as a percentage of mean daily energy demand with (a) 0\%, (b) 25\% and (c) 50\% excess energy supplied. Left axis: all $\tau<1$ month; Right axis: 1 month $\leq \tau<$ 1 year. 
a)

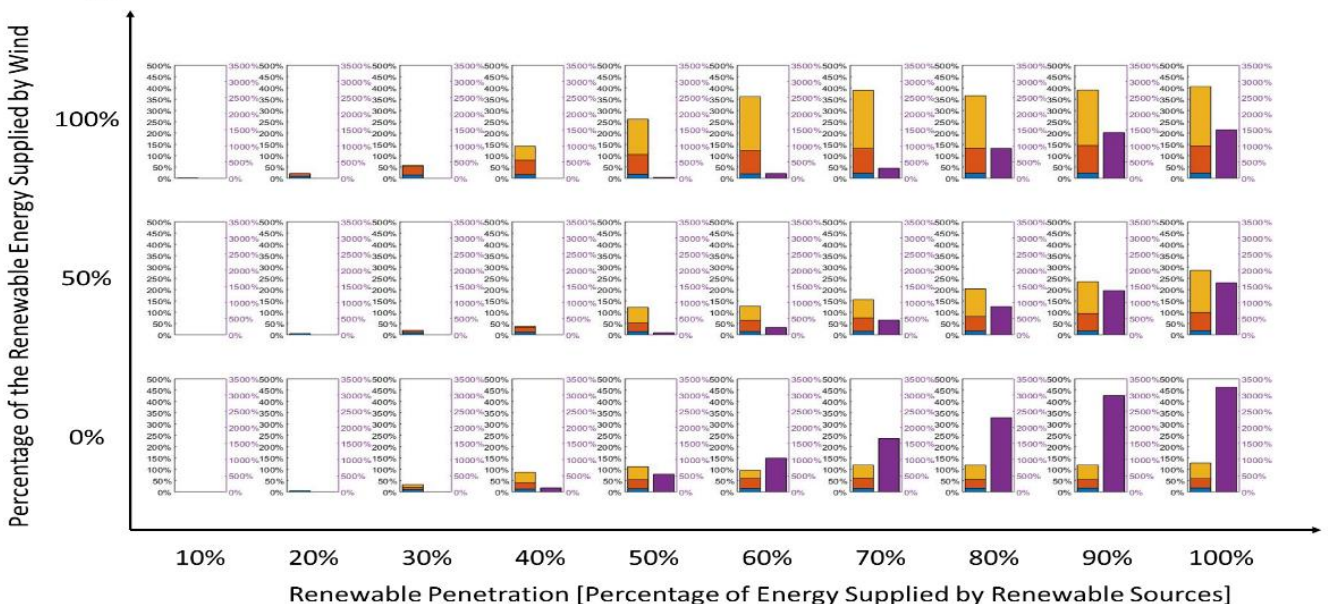

b)

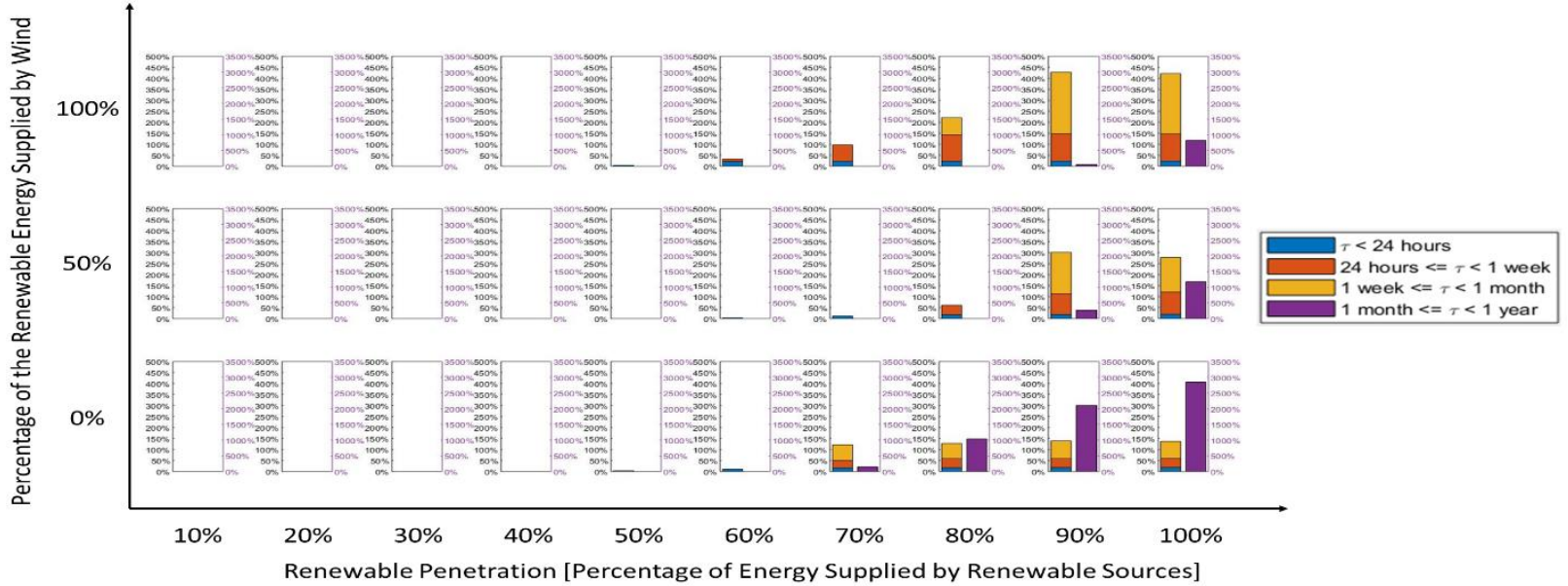

c)

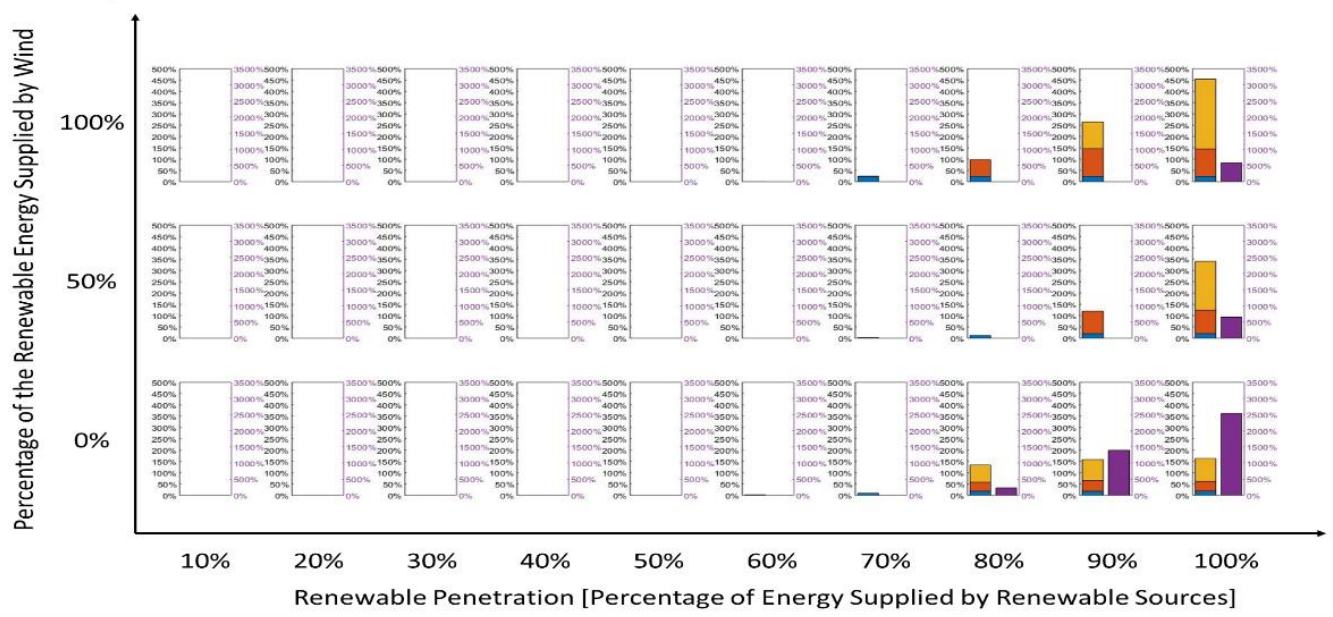

Figure 14: Most challenging location considered in the UK (Strathallan Airfield): Storage size required as a percentage of mean daily energy demand with (a) $0 \%$, (b) $25 \%$ and (c) $50 \%$ excess energy supplied. Left axis: all $\tau<1$ month; Right axis:

1 month $\leq \tau<1$ year. 


\section{Conclusions and future work}

The development of the ESRMT provides us with a tool to better understand the fundamental balancing problem of VRE integration in terms of the network flexibility (as energy storage) required. The ESRMT achieve this by using an extended pinch methodology to quantify the magnitude of storage required as a function of storage duration. Quantifying the temporal characteristics of the balancing problem provides improved understanding of the problem that needs to be managed. This additional functionality, when compared with previous methods of technical analysis, is useful for the ongoing integration of greater VRE capacity due to INDCs and the rapid cost reduction of both wind would be suitable. and solar photovoltaic [39] electricity. The ESRMT tool can be used to provide an initial estimate of additional system balancing requirements incurred due to VRE integration [40] and if time of use tariffs (or other temporal methods)

The results obtained from using the ESRMT have shown that the magnitude of storage required when increasing VRE penetration is highly dependent on the location and VRE that is supplied through wind particularly when no other flexibility method is employed. The mix of storage required, and specifically the "tipping-point" (when the storage requirement cannot be met solely by short-term storage methods), is also highly dependent on location and VRE that is supplied through wind. While considering different locations, these results largely support Weitemeyer et al.'s [26] conclusion that seasonal storage is not required below $80 \%$ VRE penetration. However, this paper shows that is not the case for certain mixes of VRE sources or more challenging locations. The size of storage required (for arbitrarily chosen time periods of storage duration) largely reflects the SMI and SDI results. However, it did show the importance of quantifying temporal aspects. For example, those scenarios considered (i.e. with a defined VRE penetration and mix) requiring a sizeable storage use (a high SMI value) and with an SDI close to 1.00 could meet their balancing requirements with only a small amount of short-term storage capacity. Excess VRE supply to mitigate the storage requirements has been shown to be vary in its effectiveness depending on the combination of VRE penetration and mix. By quantifying how the magnitude and type of required balancing varies with VRE integration, this work identifies if/when there is a need for certain technologies. These results highlight the importance of the current implementation of short-term storage (e.g. batteries) as well as the development of longer term storage methods for future deployment (e.g. power-to-gas). 
These insights and further use of the ESRMT can provide understanding and functionality to many stakeholders as power networks change to being net-zero compliant, e.g.: (i) providing policy makers with a better fundamental understanding of the balancing problem that is being faced with greater VRE integration; (ii) identifying those technologies and improvements that will be most beneficial in mitigating or managing the problem for industry; and (iii) providing an initial estimate of the domain of viable technical solutions for further techno-economic optimisation and VRE capacity credit analyses [41]. The ESRMT also allows additional context / understanding of the impact that the type of RE sources has on a network flexibility requirements when increasing the RE capacity. Overall, the ESRMT informs stakeholders about the importance of considering additional system benefits and costs associated with integrating VRE.

In addition to the functionality shown in this paper, further work using the ESRMT can quantify the mitigating potential of other flexibility methods, such as demand-side management, interconnection, reserves, and additional demand for a given location. Such analyses would not only more accurately quantify the system difficulties of integrating additional VRE capacity in existing networks, but also how hard it will be to mitigate and / or manage them. Despite the usefulness of the existing tool, the methodology and calculations presented here do have limitations. Future work should include the incorporation of non-ideal prediction of supply and demand [42], limiting the rated power and ramping of dispatchable sources, and storage efficiency. The incorporation of this functionality to the ESRMT in addition to considering other methods of flexibility (mentioned previously) will impact the magnitude and duration of storage required.

\section{Nomenclature (alphabetical order)}

\section{$\tau$}

D

DSM

E

ESRMT

GHG

$\mathrm{H}$

I

INDC

L

$\mathrm{O}$

S

SDI

SMI

$\mathrm{t}$
Storage duration [hours]

Energy vector for dispatchable supply sources [MWh]

Demand Side Management

Cumulative fraction of demand met by storage energy

Energy storage requirement modelling tool

Greenhouse Gas

Height [meters]

Energy vector for energy stored [MWh]

Intended Nationally Determined Contributions

Energy vector for load (i.e. demand) [MWh]

Energy vector for energy discharged from storage [MWh]

Energy vector for solar photovoltaic [MWh]

Storage duration index

Storage magnitude index

Time [hours] 
Maximum storage duration considered for the calculation of SMI and SDI (8760 hours)

$\mathrm{U}$

VRE

$\mathrm{W}$

$\langle W\rangle$

$\mathrm{z}$
Velocity [meters/second]

Variable renewable energy

Energy vector for wind [MWh]

Total amount of wind energy supplied [MWh] $\left(\int_{t=1}^{t}\right.$ end $\left.W d t\right)$

Roughness level (taken as 0.03 , i.e. open ground)

\section{Acknowledgements}

This work was financially supported by the Siemens-EPSRC iCASE Award (Grant number 1658940). MATLAB

software was used under the Department of Engineering Science's academic licence.

\section{References}

1. Stern, N. Towards a low-carbon future. in Resource Conference 2017. 2017. Oxford, UK.

2. $\quad$ IEA, World Energy Outlook 2018. 2018.

3. Hidalgo Gonzalez, I., et al., Addressing flexibility in energy system models, in EUR - Scientific and Technical Research series. 2015, European Commission: Joint Research Centre - Institute for Energy and Transport: Luxembourg: Publications Office of the European Union.

4. Connolly, D., et al., A review of computer tools for analysing the integration of renewable energy into various energy systems. Applied Energy, 2010. 87(4): p. 1059-1082.

5. Kondziella, H. and T. Bruckner, Flexibility requirements of renewable energy based electricity systems $-a$ review of research results and methodologies. Renewable and Sustainable Energy Reviews, 2016. 53(Supplement C): p. 10-22.

6. Zerrahn, A. and W.P. Schill, Long-run power storage requirements for high shares of renewables: review and a new model. Renewable and Sustainable Energy Reviews, 2017. 79: p. 1518-1534.

7. Lund, H., Large-scale integration of optimal combinations of $P V$, wind and wave power into the electricity supply. Renewable Energy, 2006. 31(4): p. 503-515.

8. Denholm, P. and M. Hand, Grid flexibility and storage required to achieve very high penetration of variable renewable electricity. Energy Policy, 2011. 39(3): p. 1817-1830.

9. Tuohy, A. and M. O'Malley, Wind Power and Storage, in Wind Power in Power Systems, Second Edition. 2012, John Wiley and Sons. p. 465-487.

10. Friedrich, D., Evaluation of the effect of flexible demand on the design and operation of hybrid energy systems, in Offshore Energy and Storage Symposium. 2016: Valletta, Malta.

11. Heard, B.P., et al., Burden of proof: A comprehensive review of the feasibility of $100 \%$ renewable-electricity systems. Renewable and Sustainable Energy Reviews, 2017. 76(Supplement C): p. 1122-1133.

12. Holttinen, H., et al., Methodologies to determine operating reserves due to increased wind power. IEEE Transactions on Sustainable Energy, 2012. 3(4): p. 713-723.

13. Mitra, J., Reliability-based sizing of backup storage. Power Systems, IEEE Transactions on, 2010. 25(2): p. 1198-1199.

14. Heide, D., et al., Reduced storage and balancing needs in a fully renewable European power system with excess wind and solar power generation. Renewable Energy, 2011. 36(9): p. 2515-2523.

15. Heide, D., et al., Seasonal optimal mix of wind and solar power in a future, highly renewable Europe. Renewable Energy, 2010. 35(11): p. 2483-2489.

16. Rasmussen, M.G., G.B. Andresen, and M. Greiner, Storage and balancing synergies in a fully or highly renewable pan-European power system. Energy Policy, 2012. 51: p. 642-651.

17. Initiative, C.P., Low-cost, low-carbon power systems: How to develop competitive renewablebased power systems through flexibility. 2017.

18. Blarke, M.B. and H. Lund, The effectiveness of storage and relocation options in renewable energy systems. 
Renewable Energy, 2008. 33(7): p. 1499-1507.

19. Holttinen, H., L. Söder, and E. Ela, Design and operation of power systems with large amounts of wind power: Final report, IEA WIND Task 25, Phase 4 2015-2017. 2018: VTT Technical Research Centre of Finland.

20. Brandon, N.P., et al., UK research needs in grid scale energy storage technologies. 2016, Energy SuperStore.

21. REN21, Renewables Global Futures Report: Great debates towards 100\% renewable energy. 2017, Paris: REN21 Secretariat.

22. Palizban, O. and K. Kauhaniemi, Energy storage systems in modern grids-Matrix of technologies and applications. Journal of Energy Storage, 2016. 6: p. 248-259.

23. $\quad$ Simpson, M., RESTLESS Energy Storage Database. 2016.

24. Arbon, I.A., et al., Energy storage the missing link in the UK's energy commitments. 2014, Institute of Mechanical Engineers (IMechE).

25. Hedegaard, K. and P. Meibom, Wind power impacts and electricity storage - A time scale perspective. Renewable Energy, 2012. 37(1): p. 318-324.

26. Weitemeyer, S., et al., Integration of Renewable Energy Sources in future power systems: The role of storage. Renewable Energy, 2015. 75: p. 14-20.

27. Saarinen, L., N. Dahlbäck, and U. Lundin, Power system flexibility need induced by wind and solar power intermittency on time scales of 1-14 days. Renewable Energy, 2015. 83: p. 339-344.

28. Klemeš, J.J., et al., New directions in the implementation of Pinch Methodology (PM). Renewable and Sustainable Energy Reviews, 2018. 98: p. 439-468.

29. Srivastava, S.K., Green supply - chain management: a state - of - the - art literature review. International journal of management reviews, 2007. 9(1): p. 53-80.

30. Dunn, R.F. and M.M. El - Halwagi, Process integration technology review: background and applications in the chemical process industry. Journal of Chemical Technology \& Biotechnology: International Research in Process, Environmental \& Clean Technology, 2003. 78(9): p. 1011-1021.

31. Foo, D.C.Y., State-of-the-art review of pinch analysis techniques for water network synthesis. Industrial \& Engineering Chemistry Research, 2009. 48(11): p. 5125-5159.

32. Bandyopadhyay, S., Design and optimization of isolated energy systems through pinch analysis. Asia-Pacific Journal of Chemical Engineering, 2011. 6(3): p. 518-526.

33. Nayak-Luke, R.M. and R. Bañares-Alcántara, Quantifying the relationship between renewable energy sources and electrical energy storage requirements, in Offshore Energy and Storage Symposium. 2016: Valletta, Malta.

34. MetOffice, UK Mean Wind Data, Part of the Met Office Integrated Data Archive System (MIDAS), N.B.A.D. Centre, Editor. 2006.

35. Meteotest, Meteonorm. 2018.

36. A/S, V.W.S., General specification V90 - 3.0MW 60Hz variable speed turbine. 2004, Vestas Wind Systems $\mathrm{A} / \mathrm{S}$.

37. ELEXON, Load Profiles and their use in Electricity Settlement. 2013.

38. AEMO, National Electricity Market: Data Dashboard - Aggregated Price and Demand Data, A.E.M.O. (AEMO), Editor. 1998.

39. IRENA, Renewable Power Generation Costs in 2018. 2018, International Renewable Energy Agency: Abu Dhabi.

40. Helm, D., The Carbon Crunch. Revised and updated ed. 2015, New Haven: Yale University Press.

41. Voorspools, K.R. and W.D. D'Haeseleer, An analytical formula for the capacity credit of wind power. Renewable Energy, 2006. 31(1): p. 45-54.

42. Michiorri, A., et al., Storage sizing for grid connected hybrid wind and storage power plants taking into account forecast errors autocorrelation. Renewable Energy, 2018. 117: p. 380-392. 